\title{
CT Fluid-Blood Levels in COVID-19 Intracranial Hemorrhage
}

W e read with great interest the article by Professor Jain, "Evolving Neuroimaging Findings during COVID-19," which highlighted the published coronavirus 2019 (COVID-19) literature on ischemic strokes associated with a possible virusinduced prothrombotic state. ${ }^{1}$ We agree that intracranial hemorrhage (ICH) associated with the pandemic may become more important, especially as therapeutic anticoagulation is initiated to treat thrombotic complications such as in deep vein thrombosis, pulmonary embolism, cardioembolic stroke, ischemic limb, and acute myocardial infarction. ${ }^{1-3}$ Already, CT demonstrating hemorrhagic conversion of ischemic strokes and hemorrhagic strokes composes up to one-quarter of positive neuroimaging findings. ${ }^{1,2} \mathrm{ICH}$ secondary to anticoagulation therapy is an important iatrogenic complication that radiologists need to recognize as we aid our clinician counterparts in appropriate treatment and prognostication.

We recently observed such a situation in a 64-year-old male patient who was admitted to the intensive care unit with multiorgan failure secondary to severe COVID-19 pneumonia. He required extracorporeal membrane oxygenation (ECMO) support with protocolized anticoagulation therapy to prevent circuit clotting. The team had targeted and achieved a low activated partial thromboplastin time. However, on the second day, his consciousness level deteriorated, and his pupils became dilated and unresponsive. Urgent CT revealed bihemispheric multifocal intracranial hematomas, with multiple fluid-blood levels (Figure) and general sulcal and ventricular effacement. Our patient demonstrated the characteristic neuroimaging finding of intracranial hematomas containing horizontal menisci (caused by dependent separation of heavier, hyperattenuating blood from lighter, hypoattenuating serous fluid), which has a high specificity for patients who have been treated with anticoagulants. ${ }^{4}$

While ICH is a known complication of ECMO, in this patient, it represented an unexpected turn of events despite achieving low therapeutic anticoagulation. Currently, the interaction between coagulopathy and the effect of the Severe Acute Respiratory Syndrome coronavirus 2 (SARS-CoV-2) on the vascular endothelium is not well-studied. $^{3}$ Individualized risk stratification, with possible

-- Indicates open access to non-subscribers at www.ajnr.org

http://dx.doi.org/10.3174/ajnr.A6672

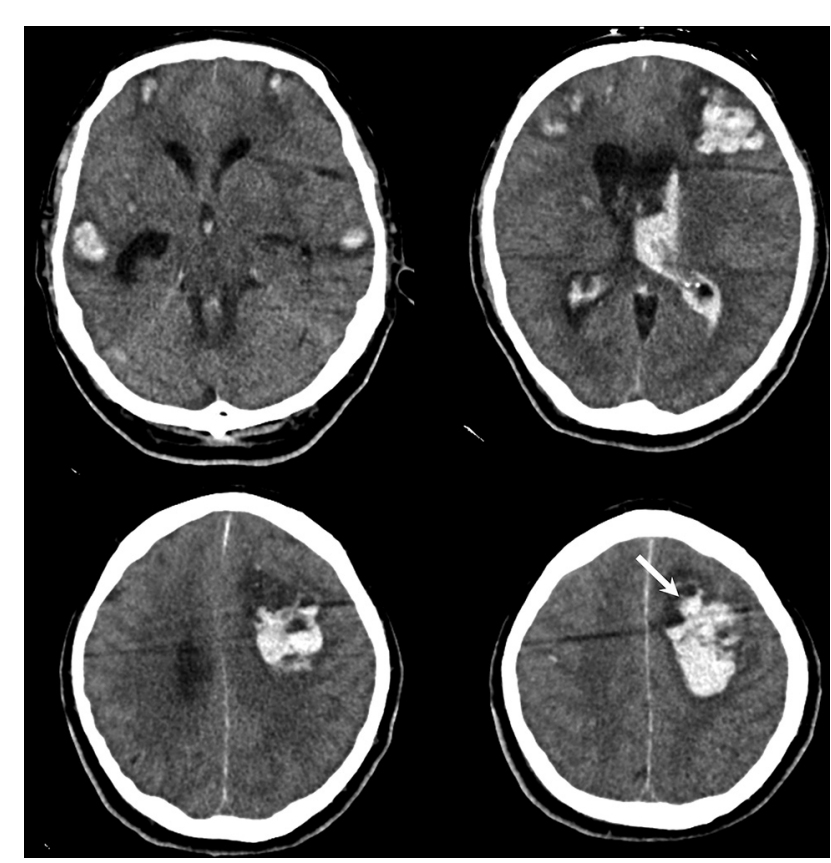

FIGURE. Unenhanced axial CT scan of the brain showing the presence of bihemispheric multifocal intracranial hematomas, with multiple fluid-blood levels (arrow) and general sulcal and ventricular effacement.

extended coagulation work-up, before and during anticoagulation therapy may be an important direction of research. Although COVID-19 infection may modify intracranial hemorrhage, it is hoped that prompt recognition and treatment of coagulopathy may help reduce mortality rates and improve clinical outcomes. ${ }^{4}$ As neuroradiologists increasingly encounter and diagnose central nervous system complications of COVID-19 infection, we should recognize the characteristic finding of fluid-blood levels within the intracranial hematoma as associated with anticoagulation.

Disclosures: Yew Woon Chia_UNRELATED: Employment: Tan Tock Seng Hospital, Singapore.

\section{REFERENCES}

1. Jain R. Evolving neuroimaging findings during COVID-19. AJNR Am J Neuroradiol 2020;41 CrossRef 
2. Dogra S, Jain R, Cao M, et al. Hemorrhagic stroke and anticoagulation in COVID-19. J Stroke Cerebrovasc Dis 2020;28:104984 CrossRef

3. Klok FA, Kruip M, van der Meer NJ, et al. Incidence of thrombotic complications in critically ill ICU patients with COVID-19. Thromb Res 2020;191:145-47 CrossRef Medline

4. Pfleger MJ, Hardee EP, Contant CF, et al. Specificity of fluid-blood levels for coagulopathy in acute intracerebral hematomas. AJNR Am J Neuroradiol 1994;15:217-23 Medline
(1) K.C.H. Lee Department of Respiratory and Critical Care Medicine Singapore General Hospital

Singapore

(1) Y.W. Chia

Department of Cardiology Tan Tock Seng Hospital Singapore

(D) N.K. Wee Department of Diagnostic Imaging

(1) E.B. Fan

Department of Hematology Tan Tock Seng Hospital Singapore
(1) T.C.C. Lim

Department of Neuroradiology, National Neuroscience Institute Duke-NUS Graduate Medical School, Singapore 